\title{
Time and age trends in urinary sugar excretion among German children and adolescents
}

\author{
Ines Perrar ${ }^{1}$, Gunter K. Kuhnle ${ }^{2}$, Thomas Remer ${ }^{1}$, Anette E. Buyken ${ }^{3}$ and Ute Alexy ${ }^{1}$ \\ ${ }^{1}$ DONALD study, Institute of Nutritional and Food Sciences -Nutritional Epidemiology, University of Bonn, Dortmund, \\ Germany, \\ ${ }^{2}$ Department of Food \& Nutritional Sciences, University of Reading, Reading, United Kingdom and \\ ${ }^{3}$ Institute of Nutrition, Consumption and Health, Faculty of Natural Sciences, University Paderborn, Paderborn, \\ Germany
}

\section{Abstract}

A high sugar intake is increasingly discussed to affect health. Preceding analyses based on dietary records, collected between 19852016, suggest a decreasing time trend in total [TS], added [AS] and free [FS] sugar intake (calculated as \% of energy intake [\%E]) since 2005 - most notably from 2010 onwards - among children and adolescents in Germany. While TS [\%E] intake decreased with age, the youngest children had the lowest AS [\%E] intake and the oldest children had the lowest FS [\%E] intake, with minor differences in the other age groups. However, self-reported data is likely introducing bias due to selective underreporting, especially of sugar-rich foods, which can distort results. Therefore, we analysed time and age trends in dietary sugar intake using urinary biomarkers.

Urinary fructose excretion (FE), sucrose excretion $(\mathrm{SE})$ and the sum of both $(\mathrm{FE}+\mathrm{SE})$ as predictive biomarkers for sugar intake was measured by UPLC-MS/MS in 997 24-h urine samples from the DONALD (Dortmund Nutritional and Anthropometric Longitudinally Designed) study cohort collected from 8.5-16.5-year-olds (239 boys, 253 girls) between 1990-2016. Time and age trends of log-transformed FE, SE and FE $+\mathrm{SE}$ were analyzed using polynomial mixed-effects regression models. Sex, creatinine and urea excretion, urine volume, total daily energy intake, overweight status, type of weekday, maternal overweight and educational status as well as maternal employment were considered as potentially confounding factors.

The mean $( \pm$ SD) FE was $26.1 \pm 20.9 \mathrm{mg} / \mathrm{d}$, SE $33.4 \pm 38.3 \mathrm{mg} / \mathrm{d}$, and FE + SE $59.6 \pm 49.1 \mathrm{mg} / \mathrm{d}$. While FE increased significantly with age (linear trend: $p=0.0147$ ), there were no significant age trends for SE and FE + SE in adjusted models. Between 1990-2016 $\mathrm{FE}$ as well as FE $+\mathrm{SE}$ decreased significantly (linear trend: $p=0.0280$ and $\mathrm{p}<0.0001$, respectively). SE showed a tendency towards a negative quadratic time trend $(\mathrm{p}=0.0574)$.

The decreasing time trend of urinary sugar excretion supports our previous observed time trends in dietary sugar intake based on dietary records among children and adolescents. In contrast, the observed age trend in dietary sugar was not confirmed by the biomarker trend analysis, suggesting increasing underreporting with age during adolescence. Although predictive biomarkers do not allow conclusions on the amount of the respective dietary intake, the combination of biomarker and dietary record data points towards the need of further public health measures to support the observed decline in sugar intake among children and adolescents in Germany.

The project is funded by the German Federal Ministry of Food and Agriculture.

\section{Conflict of Interest}

AEB is a member of the International Carbohydrate Quality Consortium (ICQC). IP, GKK, TR and UA declare that they have no conflict of interest. 